\title{
Application of Zero-Forcing Adaptive Equalization in Compensation of Fading Effect of Two-hop Wireless Link
}

\author{
Abu Sayed Md. Mostafizur Rahaman, Md. Imdadul Islam, and M. R. Amin, Member, IEEE
}

\begin{abstract}
Wireless link in mobile cellular communication system is experienced by large and small scale fading. Since the link is non line of sight (NLOS), therefore, severely affected by multipath fading. In a two-hop wireless links, each link is affected by fading and noise degrades the overall performance of the communication system. In this paper, performance of two- hop links is analyzed under Rayleigh and Nakagami-m fading environments separately for QPSK and 8-PSK modulation schemes. In this paper zero-forcing adaptive equalizer is used on two-hop wireless links to enhance the performance of the system. Here we find that zero-forcing adaptive equalizer works better for QPSK scheme and Nakagami-m fading channel compared to 8-PSK scheme and the Rayleigh fading channel.
\end{abstract}

Index Terms-Probability of symbol error, NLOS, Nakagami-m and Rayleigh fading, Coherent demodulator, MMSE and Gaussian Q function.

\section{INTRODUCTION}

The electromagnetic (EM) radio wave in wireless channel may be reflected, refracted and scattered by surrounding objects which results in multipath propagation of signals. Therefore, multiple copies of the same signal arrive at the receiving end and creates delay spread. When separation between the transmitter and the receiver is very large then mean signal strength is considered at the receiver (known as large scale propagation model) but when separation between the transmitter and the receiver is low (less than $5 \mathrm{~km}$ ) usually in an urban or suburban area, then rapid variation of the signal strength within short distance or short duration is considered (known as small scale propagation model). Under a multipath propagation environment, the amplitude and phase of a composite modulated symbol very widely and rapidly, a phenomenon known as fading. Two most important parameters of a fading channel are the coherence time and the coherence bandwidth as described in [1],[2]. A lot of parameters like: symbol period, multipath delay spread, Doppler spread, coherence time/ bandwidth, time variant or invariant property, channel gain etc. plays vital role on the performance of a wireless link as summarized in [1]-[3].

A channel may be time selective or frequency selective

Manuscript received August 3, 2011; revised November 16, 2011.

Abu Sayed Md. Mostafizur Rahaman and Md. Imdadul Islam are with the Department of Computer Science and Engineering, Jahangirnagar University, Dhaka 1342, Bangladesh. Md. Imdadul Islam works also as an adjunct professor of Electronics and Communications Engineering Department at East West University, 43 Mohakhali, Dhaka 1212, Bangladesh (e-mail: imdad@juniv.edu).

M. R. Amin is with the Electronics and Communications Engineering Department, East West University, 43 Mohakhali, Dhaka 1212, Bangladesh (e-mail: ramin@ewubd.edu). depending on the time-varying nature of the impulse response of the channel. From the autocorrelation of the channel impulse response, the channel may be classified as wide-sense stationary (WSS), uncorrelated scattering (US) or wide-sense stationary uncorrelated scattering (WSSUS) channel. The complex envelope of a modulated wave is a random variable (RV) and may follow Rayleigh, Ricean, or Nakagami-m distribution depending on the condition of the channel. For example, if there is a strong line-of-sight (LOS) between the transmitter and the receiver along with multipath fading, the distribution follows Ricean probability density function (PDF) instead of Rayleigh PDF.

Dual-hop wireless link has gained much interest in recent years because of its application in co-operative wireless networks [4]-[6]. In [7], end-to-end performance of dual-hop relaying systems is evaluated considering beam forming over Nakagami-m fading channels. The source and destination uses $N_{t}$ and $N_{r}$ number of antennas while the relay station uses only one antenna. Similar analysis is done in [8] and [9] for amply-and-forward (AF) and imperfect channel case. Both the papers plot the outage probability and bit error rate (BER) against signal-to-noise ratio (SNR). In [10], a number of relay nodes are placed randomly and the best relay is selected to observe the outage probability and the BER. The combination of Rayleigh and Riccian fading is considered in [11] for two-hop AF case. In [12], the performance of dual-hop relay link is determined for coherent and non-coherent binary modulation schemes when the relay stream is only for AF purpose. The paper considers both average power scaling and instantaneous power scaling constraints over Nakagami-m fading channel.

In this paper, adaptive equalizer is added at relay and receiving end to improve the performance of the dual-hop wireless link. The paper considers 8-PSK and QPSK schemes under Rayleigh and Nakagami-m fading environments.

The paper is organized as follows. Section II gives some review work on the theoretical analysis of adaptive equalization and then describes the model of the two-hop wireless link under fading environment. Section III gives the results, and finally, Sec. IV concludes the entire analysis.

\section{SYSTEM MODEL}

\section{A. Adaptive Equalizer}

Let us consider that the transmitted signal of an wireless communication system is $s(t)=m(t) \cos (2 \pi f t+\phi(t))$, the received signal of an $\mathrm{N}$-multipath channel can be written as [13-14]:

$$
x(t)=\sum_{n=1}^{N} \alpha_{n}(t) m(t) \cos \left(2 \pi f t+\phi(t)+\theta_{n}(t)\right),
$$


where $\alpha_{n}(t)$ is the attenuation and $\theta_{n}(t)$ is the phase rotation of the $n$-th reflected path. In most of the cases, the impulse response of the channel is found time selective, i.e., the channel is better at some selected times than other times. To neutralize the delay spread of the wireless channel, adaptive equalizer is used in the passband signal [13]-[15]. In this study, a zero-forcing adaptive equalizer circuit is used at receiver end to neutralize the fading effect. Here, QPSK and 8-PSK modulation schemes are selected to compare the symbol error probability for the case of with and without equalization.

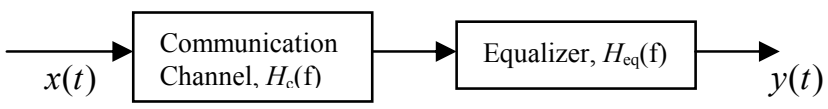

Fig. 1. Equalizer in cascade with the channel.

If any signal $x(t)$ is multiplied by a constant $k$ or delayed by an amount $t_{d}$, then the resultant signal becomes $k x\left(t-t_{d}\right)$. Such distortion is called linear distortion and linear distortion is curable in communication system. For a linear distortion channel of Fig. 1, $y(t)=k x\left(t-t_{d}\right)$. Taking Fourier transform, we have $Y(f) / X(f)=k e^{-j \omega t} d=H(f)$. In presence of distorting channel of transfer function $H_{\mathrm{c}}(f)$, received signal can be made distortionless incorporating a equalizer at the receiving end as shown in Fig. 1, where the overall transfer function of the system becomes $H_{c}(f) H_{e q}(f)=k e^{-j \omega_{d}}$, where $|H(f)|$ is called the amplitude response which is needed to be constant over the entire bandwidth (BW) of the input signal and $\theta(f)$ is the phase response and needed to be a linear function of frequency over the entire BW of the input signal for recovery of the transmitted symbol [15]-[17].

Let us consider the tapped delay finite impulse response (FIR) filter of Fig. 2, where the output signal is [18],[19]:

$$
y(t)=\sum_{k=-N}^{N} C_{k} x(t-(N+k) \tau) .
$$

Taking Fourier transform of Eq. ( 1), we have

$$
Y(f)=\sum_{k=-N}^{N} C_{k} e^{-j(N+k) \omega \tau} X(f),
$$

from which we obtain

$$
\begin{aligned}
H_{e q}(f) & =\frac{Y(f)}{X(f)} \\
& =\sum_{k=-N}^{N} C_{k} e^{-j(N+k) \omega \tau} \\
& =e^{-j N \omega \tau} \sum_{k=-N}^{N} C_{k} e^{-j k \omega \tau} .
\end{aligned}
$$

If the relation $H_{c}(f) H_{e q}(f)=k e^{-j \omega t_{d}}$ can be satisfied by adjusting the number of delay blocks and weighting factors of Eq. (1), then the distortion at the receiving end can be completely eliminated.

\section{B. Zero-Forcing Equalizer}

Taking inverse Fourier transform of

We obtain

$$
H_{e q}(f)=e^{-j N \omega \tau} \sum_{k=-N}^{N} C_{k} e^{-j k \omega \tau},
$$

$$
h_{e q}(t)=e^{-j N \omega \tau} \sum_{n=-N}^{N} C_{n} \delta(t-n \tau) .
$$

For the distorted input signal $x(t)$, the equalized output is

$$
y(t)=x(t) * h_{e q}(t)=e^{-j N \omega \tau} \sum_{n=-N}^{N} C_{n} x(t-n \tau)
$$

Taking absolute value of the output signal $y(t)$, we have

$$
|y(t)|=\sum_{n=-N}^{N} C_{n} x(t-n \tau) .
$$

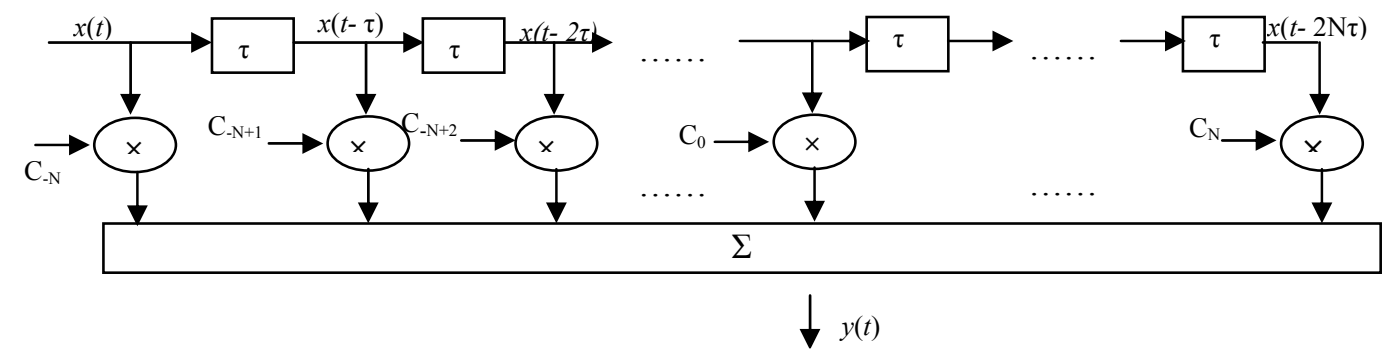

Fig. 2. Tapped delay equalizer.

In zero-forcing condition, $y(t)$ is sampled at $t=m T$ and a condition

$$
\begin{aligned}
y(m T) & =\sum_{n=-N}^{N} C_{n} x(m T-n \tau) \\
& =\delta(m)= \begin{cases}1 ; & m=0 \\
0 ; & m= \pm 1, \pm 2, \ldots, \pm N .\end{cases}
\end{aligned}
$$

Taking $T=\tau=1$,

$$
y(m)=\sum_{n=-N}^{N} C_{n} x(m-n) .
$$

We may expressed the relation of $\{y(m)\},\{x(m)\}$ and $\left\{C_{n}\right\}$ in the following matrix form:

$$
\begin{aligned}
& \text { where } \\
& \mathbf{x}=\left[\begin{array}{cccccc}
x(0) & x(-1) & x(-2) & \ldots & x(-2 N+1) & x(-2 N) \\
\vdots & \vdots & \vdots & \ddots & \vdots & \\
x(N-1) & x(N-2) & x(N-3) & \cdots & x(-N) & x(-N-1) \\
x(N) & x(N-1) & x(N-2) & \cdots & x(-N+1) & x(-N) \\
x(N+1) & x(N) & x(N-1) & \cdots & x(-N+2) & x(-N+1) \\
\vdots & \vdots & \vdots & \ddots & \vdots & \vdots \\
x(2 N) & x(2 N-1) & x(2 N-2) & \ldots & x(1) & x(0)
\end{array}\right]
\end{aligned}
$$

The dimension of the above vector $\mathbf{x}$ is $(2 N+1) \times(2 N+1)$ and that of vectors $\mathbf{y}=\left[\begin{array}{llllll}y(-2 N) & \cdots & y(0) & \cdots & y(2 N)\end{array}\right]^{T}$ and $\mathbf{c}=\left[\begin{array}{lllll}c_{-N} & \cdots & c_{0} & \cdots & c_{N}\end{array}\right]^{T}$ are $(4 N+1) \times 1$ and $(2 N+1) \times 1$ 
respectively.

The same job can be done using adaptive algorithm of minimum mean square error (MMSE). In this case, the weighting vector,

$$
\mathbf{c}=\mathbf{R}_{x x}^{-1} \mathbf{R}_{x y},
$$

where $\mathbf{R}_{x x}=\mathbf{x}^{T} \mathbf{x}$, and is called autocorrelation matrix and $\mathbf{R}_{x y}=\mathbf{x}^{T} \mathbf{y}$ is called the cross-correlation vector.

\section{Two-Hop Wireless Link}

In a dual-hop wireless communication system the sender node $\mathrm{S}$ communicates with the detector node $\mathrm{D}$ through the relay station $\mathrm{R}$ as shown in Fig. 3. Let us first concentrate on the analytical solution of the probability of symbol error, $P s$, under additive white Gaussian noise (AWGN) channel in a fading environment.

In MPSK modulation scheme, the received signal vector of coherent demodulator on $\Phi_{1}(t)-\Phi_{2}(t)$ plane is

$$
\mathbf{r}=\left[\begin{array}{l}
r_{1} \\
r_{2}
\end{array}\right] \text {, }
$$

where

$$
r_{1}=\int_{0}^{T}\left\{S_{i}(t)+n(t)\right\} \Phi_{1}(t) d t=\sqrt{E} \cos \theta_{i}+n_{1},
$$

and

$$
r_{2}=\int_{0}^{T}\left\{S_{i}(t)+n(t)\right\} \Phi_{2}(t) d t=\sqrt{E} \sin \theta_{i}+n_{2} .
$$

The signal $S_{i}(t)$ is the $i$-th modulated wave in the region $k T$ $\leq t \leq(k+1) T, E$ is the energy of a symbol, $n_{1}$ and $n_{2}$ are the noise of the in-phase and quadrature component of the received signal respectively.
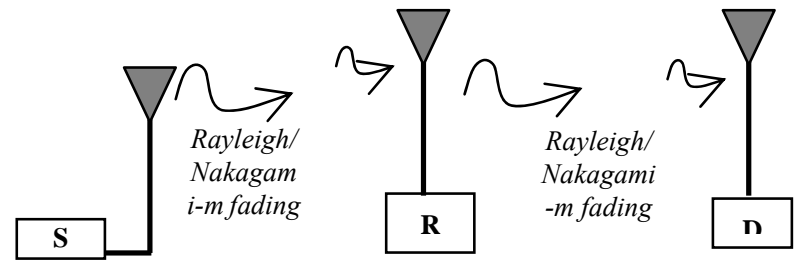

Fig. 3 Two-hop wireless link.

Taking $r_{1}=\rho \cos \hat{\theta}_{i}$ and $r_{2}=\rho \sin \hat{\theta}_{i}$, the joint PDF of $\rho$ and $\hat{\theta}_{i}$ (given $S_{i}(t)$ is transmitted) is [3]:

$$
\begin{aligned}
p\left\{\rho, \hat{\theta}_{i} \mid S_{i}(t) \text { is transmitte } d\right\} & \\
= & \frac{1}{\pi N_{0}} \exp \left[-\frac{1}{N_{0}}\left\{\rho^{2}+E-2 \rho \sqrt{E} \cos \left(\theta_{i}-\hat{\theta}_{i}\right)\right\}\right] .
\end{aligned}
$$

Integrating both side of Eq. (7) with respect to $\rho$ and taking $\varphi=\hat{\theta}_{i}-\theta_{i} \in[-\pi, \pi]$, the PDF of $\varphi$ becomes

$$
\begin{aligned}
& p\left\{\varphi \mid S_{i}(t) \text { is transmitted }\right\} \\
& =\frac{e^{-E / N_{0}}}{2 \pi}\left[1+\sqrt{\frac{\pi E}{N_{0}}}(\cos \varphi) e^{-\left(E / N_{0}\right) \cos ^{2} \varphi} G(\varphi)\right],
\end{aligned}
$$

where

$$
G(\varphi)=\left\{1+\operatorname{erf}\left(\sqrt{\frac{E}{N_{0}}} \cos \varphi\right)\right\}
$$

The probability of symbol error then can be written as [3], [13]:

$$
\begin{aligned}
P_{S} & =1-\int_{-\pi / M}^{\pi / M} p\left\{\varphi \mid S_{i}(t) \text { is transmitted }\right\} d \varphi \\
& =\frac{M-1}{M}-0.5 \operatorname{erf}\left\{\sqrt{\frac{E}{N_{0}}} \sin \left(\frac{\pi}{M}\right)\right\} \\
& -\frac{1}{\sqrt{\pi}} \int_{0}^{\sqrt{E / N_{0}}} \sin \left(\frac{\pi}{M}\right) e^{-y^{2}} \operatorname{erf}\left(y \cot \frac{\pi}{M}\right) d y,
\end{aligned}
$$

where $M>4$.

If $E / N_{0} \gg 1$, then Eq. (9) can be approximated to

$$
P_{S} \approx 2 Q\left(\sqrt{\frac{2 E}{N_{0}}} \sin \left(\frac{\pi}{M}\right)\right) .
$$

Let us now consider the generalized form of the symbol error probability of different modulation schemes using Gaussian Q function, $Q(g \sqrt{\gamma})$, where $g$ is a constant and depends on the modulation scheme and detection technique (for example, $g=\sqrt{2}$ for BPSK, $g=1$ for QPSK).

In a slow fading channel, the instantaneous SNR per bit, $\gamma=E / N_{0}$, is a time invariant RV with $\operatorname{PDF} f_{\Gamma}(\gamma)$ which depends on the particular fading environment. The average probability of error can be found for a fading channel as [2], [20]:

$$
P_{e}=\int_{0}^{\infty} P_{s}(\gamma) f_{\Gamma}(\gamma) d \gamma
$$

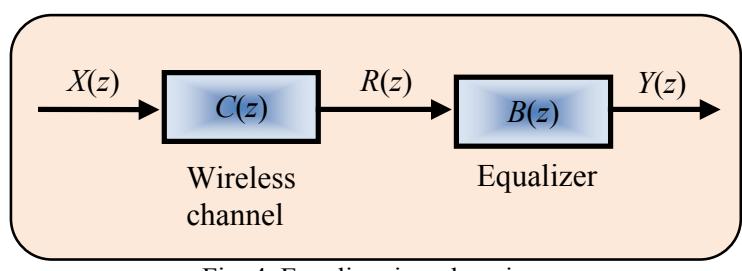

Fig. 4. Equalizer in z-domain.

In this paper we consider Rayleigh and Nakagami-m fading channels, their PDFs are:

$$
\begin{gathered}
\left.f_{\Gamma}(\gamma)\right|_{\text {Rayleigh }}=\frac{1}{\gamma_{\text {avg }}} e^{-\gamma / \gamma_{\text {avg }},} \\
\left.f_{\Gamma}(\gamma)\right|_{\text {Nakagami-m }}=\frac{m^{m} \gamma^{m-1}}{\gamma_{\text {avg }}^{m} \Gamma(m)} e^{-m \gamma / \gamma_{\text {avg }}},
\end{gathered}
$$

where $m$ is the Nakagami-m fading parameter which ranges from $1 / 2$ to $\infty$.

Let the transfer function of the wireless channel and the equalizer to be $C(\mathrm{z})$ and $B(\mathrm{z})$ respectively, as shown in Fig. 4. The $\mathrm{z}$-transform of the received signal is then

$$
Y(z)=X(z) C(z) B(z),
$$

from which we obtain the overall transfer function

$$
H(z)=\frac{Y(z)}{X(z)}=C(z) B(z) .
$$

To eliminate intersymbol interference (ISI), we need to impose the following requirement [18],[19]: $Y(z)=X(z)$, that is $H(\mathrm{z})=C(\mathrm{z}) B(\mathrm{z})=1$, which implies that

$$
c(n) * b(n)=\delta(n) \text {, }
$$


or

$$
\sum_{k=-N}^{N} b(k) c(n-k)= \begin{cases}1, & n=0 \\ 0, & n \neq 0 .\end{cases}
$$

Let the impulse response of the channel be

$$
c(t)=\sum_{k=0}^{m} a_{k} \delta(t-k T) .
$$

The z-transform of $c(t)$ gives

$$
C(z)=\sum_{k=0}^{m} a_{k} z^{-k} .
$$

The transfer function of the equalizer is $H(\mathrm{z})=C(\mathrm{z}) B(\mathrm{z})=1$, thus we obtain

$$
\begin{aligned}
B(z) & =\frac{1}{C(z)} \\
& =\left(\sum_{k=0}^{m} a_{k} z^{-k}\right)^{-1} \\
& =b_{0}+b_{1} z^{-1}+b_{2} z^{-2}+\ldots \quad \ldots \quad \ldots \quad .
\end{aligned}
$$

In the presence of additional noise $n_{n}$ at the end of $n$-th symbol interval at the effective channel output, the variance of noise will be

$$
\begin{aligned}
\sigma_{v}^{2} & =\sum_{k=0}^{\infty}\left(b_{k}\right)^{k} n_{n-k} \\
& =\frac{N_{0}}{2} \sum_{k=0}^{\infty}\left(b_{k}\right)^{k} .
\end{aligned}
$$

Therefore, the probability of bit error is

$$
P_{b}=Q\left(\sqrt{\frac{E_{b}}{\sigma_{v}^{2}}}\right) .
$$

If the transmit power at sender and repeater stations are $P_{1}$ and $P_{2}$ with fading amplitudes $\left|h_{S R}\right|$ and $\left|h_{R D}\right|$ respectively, then SNR of S-R and R-D links are[11]-[13], [20]:

$$
\gamma_{1}=\frac{\left|h_{S R}\right|^{2}}{N_{0}} P_{1} \text {, }
$$

and

$$
\gamma_{2}=\frac{\left|h_{R D}\right|^{2}}{N_{0}} P_{2},
$$

where $N_{0} / 2$ is the two-sided power spectral density (psd) of the AWGN. The equivalent SNR of the two-hop link is

$$
\gamma_{e q}=\frac{\gamma_{1} \gamma_{2}}{\gamma_{1}+\gamma_{2}+c}
$$

where $c=1$ for exact value of $\gamma$ and $c=0$ for approximate value of $\gamma$ at higher SNR.

\section{RESULTS}

Let us consider an adaptive equalizer of 7 taps and a received distorted set of pulse samples:

$$
\{\mathrm{x}(\mathrm{m})\}=\left[\begin{array}{llll}
0.0218 & -0.0564 & 0.1413 & 0.7000 \\
& -0.1820 & 0.0325 & 0.0230
\end{array}\right]
$$

We will find the weighting vector of the zero-frocing adaptive equalizer. For 7 taped filter, the dimension of matrix $\mathbf{x}$ is $2 N+1 \times 2 N+1=7 \times 7$ and is given as,

$\begin{array}{rrrrrrr}\mathbf{X}= & & & & & & \\ 0.7000 & -0.1820 & 0.0325 & 0.0230 & 0.0000 & 0.0000 & 0.0000 \\ 0.1413 & 0.7000 & -0.1820 & 0.0325 & 0.0230 & 0.0000 & 0.0000 \\ -0.0364 & 0.1413 & 0.7000 & -0.1820 & 0.0325 & 0.0230 & 0.0000 \\ 0.0218 & -0.0564 & 0.1413 & 0.7000 & -0.1820 & 0.0325 & 0.0230 \\ 0.0000 & 0.0218 & -0.0564 & 0.1413 & 0.7000 & -0.1820 & 0.0325 \\ 0.0000 & 0.0000 & 0.0218 & -0.0564 & 0.1413 & 0.7000 & -0.1820 \\ 0.0000 & 0.0000 & 0.0000 & 0.0218 & -0.0564 & 0.1413 & 0.7000\end{array}$

Applying MATLAB 9.x, we get the auto-correlation matrix,

$$
\begin{aligned}
& \mathbf{R}_{\mathrm{xx}}= \\
& \begin{array}{lllllll}
0.5118 & -0.0349 & -0.0254 & 0.0426 & -0.0019 & -0.0001 & 0.0005
\end{array} \\
& \begin{array}{lllllll}
-0.0349 & 0.5467 & -0.0436 & -0.0436 & 0.0462 & -0.0026 & -0.0006
\end{array} \\
& \begin{array}{lllllll}
-0.0254 & -0.0436 & 0.5478 & -0.0429 & -0.0436 & 0.0462 & -0.0026
\end{array} \\
& \begin{array}{lllllll}
0.0426 & -0.0436 & -0.0429 & 0.5483 & -0.0429 & -0.0436 & 0.0462
\end{array} \\
& \begin{array}{lllllll}
-0.0019 & 0.0462 & -0.0436 & -0.0429 & 0.5479 & -0.0416 & -0.0466
\end{array} \\
& \begin{array}{lllllll}
-0.0001 & -0.0026 & 0.0462 & -0.0436 & -0.0416 & 0.5447 & -0.0337
\end{array} \\
& \begin{array}{lllllll}
0.0005 & -0.0006 & -0.0026 & 0.0462 & -0.0466 & -0.0337 & 0.5247
\end{array}
\end{aligned}
$$

The cross-correlation vector,

$$
\begin{array}{r}
\mathbf{R}_{\mathrm{xy}}= \\
0.0218 \\
-0.0564 \\
0.1413 \\
0.7000 \\
-0.1820 \\
0.0325 \\
0.0230
\end{array}
$$

The weighting vector is

$$
\begin{array}{r}
\mathbf{W}= \\
-0.0475 \\
0.0427 \\
0.3352 \\
1.3098 \\
-0.2049 \\
0.1155 \\
-0.0806
\end{array}
$$

Fig. 5 shows the 7 samples at both input and output of the filer where the interfering components at output is found negligible.

Taking the co-efficients of the impulses of the channel, $a_{0}$ $=0.25, a_{1}=0.134, a_{2}=0.175, a_{3}=0.0231, a_{4}=0.12$, we get the transfer function of the equalizer as

$$
H(z)=3.0769-1.268 z^{-1}-1.1337 z^{-2}+0.931 z^{-3}-0.8196 z^{-4} \text {. }
$$

Therefore, the variance of the noise is

$$
\sigma_{v}^{2}=\left(b_{0}+b_{1}+b_{2}^{2}+b_{3}^{3}+b_{4}^{4}\right) \frac{N_{0}}{2}=4.353 \frac{N_{0}}{2} .
$$

The probability of bit error of both 8-PSK and QPSK schemes under Rayleigh and Nakagami-m fading is plotted in Fig. 6 for two-hop wireless link. The Fig. 6 compares the performance of the two-hop link with and without zero-forcing equalization.

We know that the BER of a single-hop link is always better than that of two-hop link case for any type of modulation scheme because of low SNR experienced by the equivalent link of two-hop system. In this section we only consider the comparison of two-hop wireless link under two modulation schemes, viz., QPSK and 8-PSK, and inclusion of zero-forcing equalizer at the repeater and receiving end. We observe from Fig. 6 that the BER of QPSK modulation is found much lower than that of 8-PSK case because of the signal space of the constellation diagram. Finally, the BER of the Nakagami-m fading is found better than that of Rayleigh fading case for both type of the modulation schemes. In Nakagami-m fading, the parameter $\mathrm{m}$ is related to the number 
of LOS paths whereas in Rayleigh fading case, there is no strong LOS path, hence performance of Nakagami-m fading is better than the Rayleigh fading channel as is easily seen from Fig. 6.

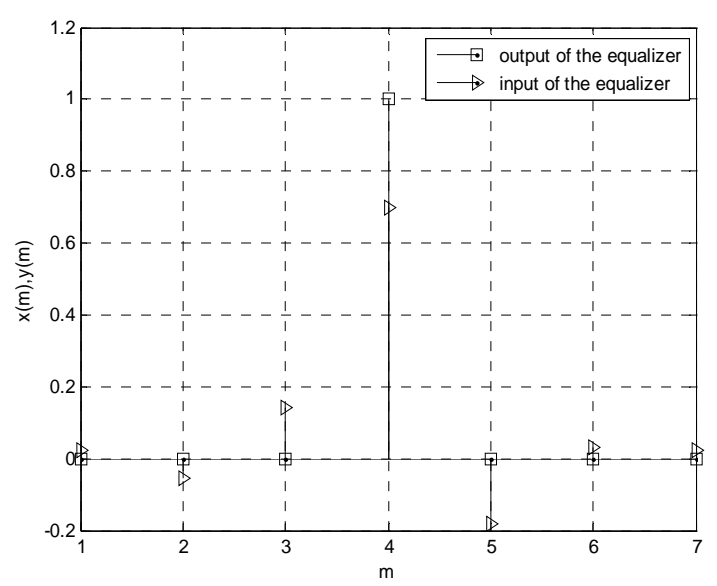

Fig. 5. Compensation of multipath affect on wireless link.

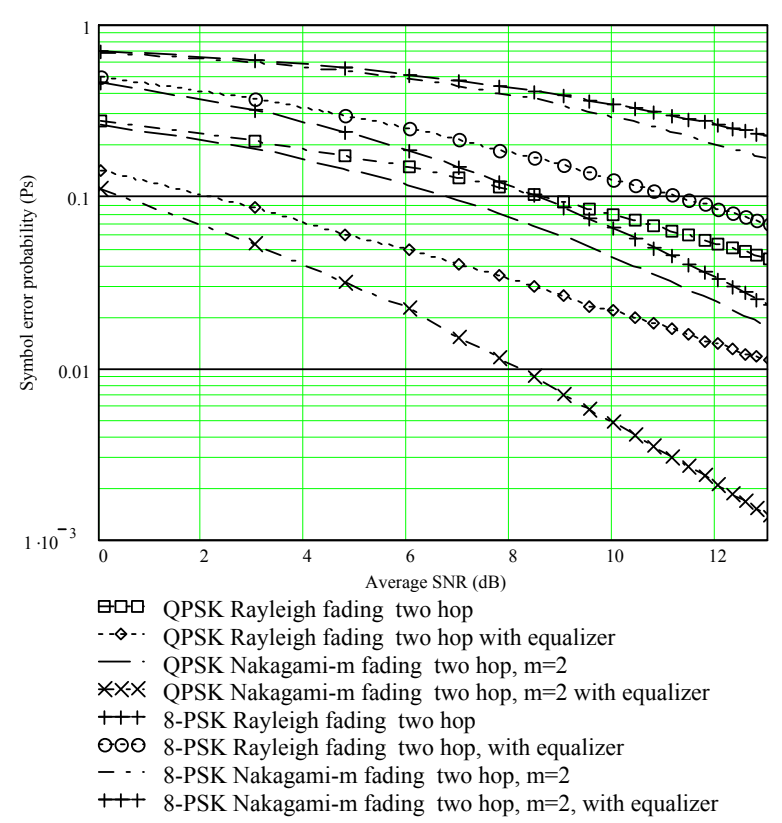

Fig. 6. Comparison of BER of two-hoped wireless link under zero forcing adaptive equalizer.

The BER of both the modulation schemes is improved with incorporation of the zero-forcing adaptive equalizer as is visualized from Fig. 6. The impact of zero-forcing equalizer is much more prominent for the QPSK scheme than that of 8-PSK scheme at the same time the zero-forcing equalizer performs well at higher SNR. Finally, the equalizer works better for the Nakagami-m fading channel compared to that of the Rayleigh fading channel.

\section{CONCLUSION}

In this paper, we have incorporated zero-forcing adaptive equalizer at the repeater and the receiving end to enhance performance of the two-hop wireless links. Zero-forcing adaptive equalizer improves performance of the two-hop links for both QPSK and 8-PSK modulation schemes under the Rayleigh and Nakagami-m fading environments. It has also been observed that the performance of the Nakagami-m model is better than that of the Rayleigh fading model. In this study, a single antenna scheme is used at the sender, the repeater and the receiving end. We have the scope to consider the multiple-input single-output (MISO) link between the transmitter and the repeater and single-input multiple-output (SIMO) between the repeater and the receiving end. It should be further mentioned here that the adaptive equalizer can be incorporated also with orthogonal space diversity at stage-1 and maximal ratio combining (MRC) at stage-2 to observe the performance of multiple antenna at the sender and the receiver with single antenna at the repeater. The work along this line is in progress and will be reported elsewhere.

\section{APPENDIX A}

The output of the equalizer at a discrete instant of time $n$ is

$$
y(n)=\sum_{k=-N}^{N} w_{k} x(n-k) .
$$

The error signal is defined as $e(n)=z(n)-y(n)$. The mean square error is

$$
\begin{aligned}
& \zeta= E\left[e(n) e^{*}(n)\right] \\
&=E\left[|e(n)|^{2}\right] \\
&=E\left[\left|z(n)-\sum_{k=-N}^{N} w_{k} x(n-k)\right|^{2}\right] \\
&=E\left[|z(n)|^{2}\right]-\sum_{k=-N}^{N} w_{k}^{*} E\left[x(n-k) z^{*}(n)\right] \\
& \quad-\sum_{k=-N}^{N} w_{k} x^{*}(n-k) z(n) \\
& \quad+\sum_{k=-N}^{N} \sum_{i=-N}^{N} w_{k}^{*} w_{i} E\left[x(n-k) x^{*}(n-i)\right] \\
& \quad \sigma_{z}^{2}-\sum_{k=-N}^{N} w_{k}^{*} p(-k)-\sum_{k=-N}^{N} w_{k} p^{*}(k) \\
& \quad+\sum_{k=-N}^{N} \sum_{i=-N}^{N} w_{k}^{*} w_{i} \gamma(i-k),
\end{aligned}
$$

where

$$
\begin{aligned}
& \gamma(i-k)=E\left[x(n-k) x^{*}(n-i)\right], \\
& p(-k)=E\left[x(n-k) z^{*}(n)\right], \\
& p^{*}(k)=E[x(n-k) z(n)] .
\end{aligned}
$$

We also have,

$$
\zeta=\sigma_{z}^{2}-W^{H} P-P^{H} W+W^{H} R W,
$$

where

$$
\begin{aligned}
& R=R_{x x}, P=R_{n z}, \\
& \nabla \zeta=-2 p+2 W_{0} R=0, \\
& W_{0}=R^{-1} P=R_{x x}^{-1} R_{n z}=\beta .
\end{aligned}
$$

\section{REFERENCES}

[1] Throdore S. Rapaport, "Wireless communications principles and practice", Pearson Education, $2^{\text {nd }}$ edition.

[2] Simon Haykin, Michael Moher, "Modern Wireless Communications", Pearson Education, 2005. 
[3] Fuqin Xiong, "Digital Modulation Techniques", ARTECH HOUSE INC. 685 Canton Street, Norwood, MA 02062, 2000.

[4] J. N. Laneman, D. N. C. Tse, and G. W. Wornell, "Cooperative diversity in wireless networks efficient protocols and outage behaviour", IEEE Trans. Inf. Theory, vol. 50, no. 12, pp. 3062-3080, 2004.

[5] J. N. Laneman and G. Wornell, "Energy-efficient antenna sharing and relaying for wireless networks", in Proc. Wireless Commun. NetworkingConf. vol. 1, pp. 3062-3080, 2000.

[6] H. A. Suraweera, G. K. Karagiannidis, "Closed-Form Error Analysis of the Non-identical Nakagami-m fading Channel", IEEE Comm. Lett., vol. 12, no. 4, pp. 259-261, April 2008.

[7] D. B. da Costa and S. Assa, "Co-operative dual-hop relaying system with beamforming over Nakagami-m fading channels", IEEE Trans. Wireless Commun., vol. 8, no.8, pp.3950-3954, 2009.

[8] N. S. Ferdinand and N. Rajatheva, "Performance analysis of imperferect channel estimation in MIMO two-hop fixed gain relay network with beamformer", IEEE Comm. Lett., vo. 15, no. 2, pp 208-2010, Feb. 2011

[9] H. Q. Ngo, Tony Q. S. Quek and H. Shin, "Random coding error exponent for dual-hop Nakagami-m fading channels with amply and forward relaying", IEEE Commun. Lett., vol. 13, No. 11, pp. 823-825, Nov. 2009.

[10] A. Nessa, Q. Yang and K. Kwak, "Performance analysis of two-hop co-operative MIMO transmission with best relay selection in Rayleigh fading channel", The International Arab J. Info. Technol., vol. 8, no. 1, pp. 9-15, Jan. 2011.

[11] H. A. Suraweera, R. H.Y. Louie, Y. Li, G. K. Karagiannidis and B. Vucetic, "Two Hop Amplify-and-Forword Transmission in mixed Rayleigh and Ricean Channels", IEEE Commun. Lett., vol. 13, no. 4, pp. 227-229, April 2009.

[12] S. Nath Datta and S. Chakrabarti, "Unified Error Analysis of Dual-Hop Relay Link in Nakagami-m Fading Channel", IEEE Commun. Lett., vol 14, no. 10, pp. 897-899, Oct. 2010.

[13] Zhiwei Zebg, "Digital Communication via multi-path fading Channel", Cpre537x Final Project, pp. 17-22, Nov. 2000.

[14] X. Tang, M. S. Alounini and A. Goldsmith, " Effect of channel estimation error on M-QAM BER performance in Rayleigh fading", IEEE Trans. Commun., vol. 47, no. 12, pp. 1856-64, Dec. 1999.

[15] K. Banovic, A. R. Esam and M. A. S. Khalid,, "A Novel RadiusAdjusted Approach for Blind Adaptive Equalization", IEEE Signal Proc. Lett., vol. 13, no. 1, Jan. 2006.

[16] R. A. Valenzuela, "Performance of Adaptive Equalization for Indoor Radio Communications", IEEE Trans. Commun., vol. 31, no. 3, pp. 291-293, March 1989.

[17] C. R. Johnson Jr., "Admissibility in blind adaptive channel equalization”, IEEE Control System, vol. 11, no. 1, pp. 3-15, Jan. 1991.

[18] Simon Haykin, "Adaptive Filter Theory", Fourth Edition, Pearson Education, ISBN 81-7808-565-8, pp. 231-237, pp. 345-48, pp. 466-485.

[19] Salma Ikki, Mohamed H. Ahmed, "Performance Analysis of Cooperative Diversity Wireless Networks over Nakagami-m Fading Channel”, IEEE Commun. Lett., vol. 11, no. 4, pp. 334-336, April 2007.

[20] Md. Imdadul Islam, Md. Ariful Islam, Nur Mohammad, Mahbubul Alam, and M.R. Amin, "Comparison of LMS and FDAF Algorithms in Equalization of Fading Channel", IACSIT International J. Eng. Technol., vol.3, no.1, pp.16-21, Feb. 2011.

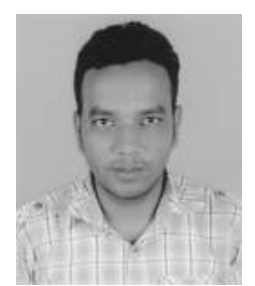

Abu Sayed Md. Mostafizur Rahaman received his B.Sc. degree in Electronics and Computer Science, from Jahangirnagar University, Savar, Dhaka, Bangladesh in 2003 and M.Sc. degree from Stuttgart University at Stuttgart, Germany in Information Technology (INFOTECH) in the branch of Embedded System Engineering in 2009. Since 2004, he is a working as an assistant professor at the Department of Computer Science and Engineering of Jahangirnagar University, Savar, Dhaka, Bangladesh. He is currently pursuing his Ph.D. at the department of Computer Science and Engineering, Jahangirnagar University, Dhaka. Currently his research focuses on telecommunication, embedded and parallel systems, reconfigurable computing, multicore architecture and software engineering.

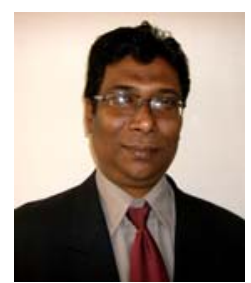

Md. Imdadul Islam has completed his B.Sc. and M.Sc Engineering in Electrical and Electronic Engineering from Bangladesh University of Engineering and Technology, Dhaka, Bangladesh in 1993 and 1998 respectively and has completed his Ph.D degree from the Department of Computer Science and Engineering, Jahangirnagar University, Dhaka, Bangladesh in the field of network traffic engineering in 2010. He is now working as a Professor at the Department of Computer Science and Engineering, Jahangirnagar University, Savar, Dhaka, Bangladesh. Previously, he worked as an Assistant Engineer in Sheba Telecom (Pvt.) LTD (A joint venture company between Bangladesh and Malaysia, for Mobile cellular and WLL), from Sept.1994 to July 1996. He has a very good field experience in installation of Radio Base Stations and Switching Centers for WLL. His research field is network traffic, wireless communications, wavelet transform, OFDMA, WCDMA, adaptive filter theory, ANFIS and array antenna systems. He has more than hundred research papers in national and international journals and conference proceedings.

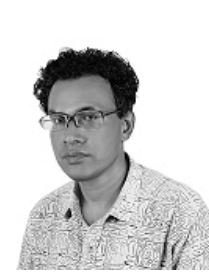

M. R. Amin received his B.S. and M.S. degrees in Physics from Jahangirnagar University, Dhaka, Bangladesh in 1984 and 1986 respectively and his Ph.D. degree in Plasma Physics from the University of St. Andrews, U. K. in 1990. He is a Professor of Electronics and Communications Engineering at East West University, Dhaka, Bangladesh. He served as a Post-Doctoral Research Associate in Electrical Engineering at the University of Alberta, Canada, during 1991-1993. He was an Alexander von Humboldt Research Fellow at the Max-Planck Institute for Extraterrestrial Physics at Garching/Munich, Germany during 1997-1999.

He was awarded the Commonwealth Postdoctoral Fellowship in 1997. Besides these, he has also received several awards for his research, including the Bangladesh Academy of Science Young Scientist Award for the year 1996 and the University Grants Commission Young Scientist Award for 1996. His current research fields are wireless communications and networks and also nonlinear plasma dynamics. He is a member of the IEEE. 